\title{
Birth order of twins and risk of perinatal death related to delivery in England, Northern Ireland, and Wales, 1994- 2003: retrospective cohort study
}

\section{Gordon C S Smith, professor of obstetrics and gynaecology, ${ }^{1}$ Kate M Fleming, senior data analyst, ${ }^{2}$ lan R White, senior scientist ${ }^{3}$}

\section{${ }^{1}$ Department of Obstetrics and Gynaecology, Cambridge University, Box 223, The Rosie Hospital, Cambridge CB2 $2 \mathrm{QQ}$ \\ ${ }^{2}$ Confidential Enquiry into Maternal and Child Health, London NW1 5SD \\ ${ }^{3}$ Medical Research Council Biostatistics Unit, Institute of Public Health, Cambridge CB2 2SR \\ Correspondence to: G C S Smith gcss2@cam.ac.uk}

doi: 10.1136/bmj.39118.483819.55

\section{ABSTRACT}

Objective To determine the effect of birth order on the risk of perinatal death in twin pregnancies.

Design Retrospective cohort study.

Setting England, Northern Ireland, and Wales, 19942003.

Participants 1377 twin pregnancies with one intrapartum stillbirth or neonatal death from causes other than congenital abnormality and one surviving infant.

Main outcome measures The risk of perinatal death in the first and second twin estimated with conditional logistic regression.

Results There was no association between birth order and the risk of death overall (odds ratio 1.0, 95\% confidence interval 0.9 to 1.1$)$. However, there was a highly significant interaction with gestational age ( $\mathrm{P}<0.001)$. There was no association between birth order and the risk of death among infants born before 36 weeks' gestation but there was an increased risk of death among second twins born at term $(2.3,1.7$ to $3.2, P<0.001)$, which was stronger for deaths caused by intrapartum anoxia or trauma (3.4, 2.2 to 5.3). Among term births, there was a trend $(P=0.1)$ towards a greater risk of the second twin dying from anoxia among those delivered vaginally (4.1, 1.8 to 9.5$)$ compared with those delivered by caesarean section $(1.8,0.9$ to 3.6$)$.

Conclusions In this cohort, compared with first twins, second twins born at term were at increased risk of perinatal death related to delivery. Vaginally delivered second twins had a fourfold risk of death caused by intrapartum anoxia.

\section{INTRODUCTION}

Though vaginal delivery of a second twin is recognised as a time of obstetric risk, we do not know whether second twins are at increased risk of perinatal death. Many studies on the association between birth order and the risk of death have methodological flaws, specifically, the failure to identify deaths truly related to delivery, the failure to use paired statistical tests to compare the outcome of first and second twins, and the failure to stratify analyses by gestational age. ${ }^{1}$ An analysis of nationally collected data on pregnancy and perinatal death from Scotland in 1992 and 1997 showed a significantly increased risk of intrapartum stillbirth or neonatal death among second twins born at term, but included only nine such deaths. ${ }^{2} \mathrm{~A}$ followup study of the same data source over a more prolonged period (1985 to 2001) showed a similar association but included only 36 deaths at term. ${ }^{3}$ A subsequent analysis of US data found no variation in the risk of neonatal death related to birth order among twins and concluded that the increased perinatal mortality among second twins was "merely an artefact of mortality comparisons." ${ }^{4}$ Data from multiple sources, however, indicate an increased risk of morbidity for the second twin at term: a large scale study from Sweden found that second twins had a fourfold risk of an Apgar score $<7$ at five minutes, ${ }^{5}$ and a recent analysis of data from Nova Scotia found a threefold risk of morbidity for the second twin delivered vaginally but no excess risk for those delivered by planned caesarean section. ${ }^{6}$ It remains unclear, therefore, whether attempted vaginal delivery of the second twin at term is associated with an increased risk of perinatal death. We studied the association between birth order and the risk of perinatal death among twin pregnancies in England, Northern Ireland, and Wales, 1994-2003.

\section{METHODS}

Data on perinatal death in England, Northern Ireland, and Wales have been collected nationally since 1994 . This is currently coordinated by the Confidential Enquiry into Maternal and Child Health (CEMACH) and had previously been done by the Confidential Enquiry into Stillbirths and Deaths in Infancy (CESDI). A network of local clinicians and health professionals at hospital level notify regional offices of perinatal deaths. Coroners' officers, child health systems, and congenital anomaly registers also report deaths. Regional managers then code the death according to the information provided, which includes autopsy results (if performed). From 1995, data from England and Wales were linked to the Office for National Statistics' registry of death certifications, ensuring 100\% ascertainment of all registered stillbirths and neonatal deaths. In 1994, the dataset was about $95 \%$ complete when compared with national 
death registrations. The death registry was compiled nationally at the inquiry's central office in London, and the CEMACH database includes all records obtained by CESDI. We used the registry of deaths for 1994-2003 for our analyses.

The data source included only information on deaths. We obtained the total number of twin births over the period of time from the Office of National Statistics and the Northern Ireland Statistics and Research Agency. However, these lack any detailed obstetric data. Hence, we had no information from cases where both twins survived and, in cases where only one twin died, we had no information on the characteristics of the survivor. We could still study the association between birth order and the risk of death, however, as statistical methods for comparison of a dichotomous outcome (such as death) in pairs uses only those instances that are discordant for the outcome of interest (see below and elsewhere for review ${ }^{1}$ ). Our analyses with this study design, however, were limited to the relative risk of death for the second twin referent to the first and determination of whether the relative risk of death for the second twin varies in relation to any characteristic common to both, such as gestational age and maternal characteristics.

We classified events using a series of fields in the available database. Firstly, we classified deaths as stillbirth, early neonatal death, and late neonatal death. A further field documented whether death of the infant took place before or after the onset of labour or whether the time of death was unknown. Intrapartum stillbirth was defined as a birth when the infant was born showing no signs of life and the death was documented as occurring during labour. Neonatal death was defined as death of a liveborn infant within the first four weeks of life. We classified cause of death according to a modified version of the Wigglesworth system into one of nine categories: congenital defect/ malformation; unexplained antepartum fetal death; death from intrapartum "asphyxia," "anoxia," or "trauma"; immaturity; infection; other specific causes; injury or non-intrapartum trauma; sudden infant death; and unclassifiable. ${ }^{7}$ We excluded those deaths where the cause was stated to be a lethal or severe congenital abnormality and classified all other intrapartum stillbirths and neonatal deaths as perinatal deaths related to delivery. We also analysed anoxic deaths as a subgroup of these events, which we defined as those where the cause was classified as "death from intrapartum asphyxia, anoxia, or trauma." As the study used wholly anonymised data and data collection was part of a national clinical audit, we did not require individual consent.

\section{Statistics}

We used conditional logistic regression to estimate the odds ratio of death for the second twin referent to the first. The method ignores concordant pairs (that is, where both twins survived or both twins died) and is therefore appropriate in a dataset containing data on deaths only. We tested for interactions between birth order and both maternal and obstetric characteristics with interaction terms and assumed significance at $\mathrm{P}<0.05$. The characteristics tested for interaction were gestational age, maternal age (expressed as a continuous variable), maternal ethnicity (white versus all others), and method of delivery. Interactions were expressed as odds ratios with 95\% confidence intervals. The latter illustrates the power of the study to exclude a given degree of effect modification and is preferable to post hoc power calculations. ${ }^{8}$ All statistical analysis was performed with Stata version 8.2 (StataCorp, College Station, TX).

\section{RESULTS}

The database had records for 5758 twin pregnancies in 1994-2003 where death of one or both infants was recorded. In 4221 of these, one infant died and the other survived. From these, we excluded eight records $(0.2 \%)$ that did not document birth order, $55(1.3 \%)$ where the gestational age at birth was missing, 450 $(10.7 \%)$ where delivery was before 24 weeks, 178 (4.7\%) where a therapeutic abortion was performed, $1975(46.8 \%)$ where death was classed as stillbirth and the timing of fetal death was before labour or unknown, and $752(17.8 \%)$ where the infant had a lethal or severe congenital abnormality. A total of 2844 records $(67.4 \%)$ had one or more of these exclusions, leaving a study group of 1377 in which there was one intrapartum stillbirth or neonatal death but the other twin survived. The table shows the characteristics of this group (table). Over the same period of time, the Office for National Statistics documented a total of 96116 certified twin pregnancies in England and Wales and the Northern Ireland Statistics and Research Agency recorded 3482, giving a total of 99598 twin pregnancies as our denominator. Further details of the denominator, such as gestational age at birth and method of delivery, were not available.

Birth order was not associated with the overall risk of perinatal death related to delivery: the odds ratio for the second twin was 1.0 (95\% confidence interval 0.9 to 1.1). There were no significant interactions between birth order and maternal age (odds ratio for interaction $0.9,0.7$ to $1.1, \mathrm{P}=0.2)$ or white ethnicity $(1.2,0.9$ to 1.5 , $\mathrm{P}=0.2$ ). There was, however, a highly significant interaction with gestational age $(\mathrm{P}<0.001)$. There was no association between birth order and the risk of death among infants born before 36 weeks' gestation (fig 1), but there was an increased risk of death among second twins born at term $(2.3,1.7$ to $3.2, \mathrm{P}<0.001)$. When we confined the analysis to deaths caused by anoxia, there was a weak association with being a second twin for all births $(1.4,1.1$ to $1.8, \mathrm{P}=0.02)$. Again, there was a highly significant interaction with gestational age $(\mathrm{P}<0.001)$. When we stratified by gestational age, we found no association between birth order and the risk of death caused by anoxia before 36 weeks (fig 1) but a strong association for births at and beyond 36 weeks (3.4, 2.2 to 5.3$)$.

We then assessed the risk of perinatal death related to delivery among second twins born at term in 


\section{Characteristics of the study cohort. Figures are numbers (percentage) unless stated otherwise}

\begin{tabular}{|c|c|}
\hline & Data \\
\hline \multicolumn{2}{|l|}{ Maternal age (years): } \\
\hline Median (IQR) & $29(25-33)$ \\
\hline Missing & $55(4.0)$ \\
\hline \multicolumn{2}{|l|}{ Ethnicity: } \\
\hline White & $1118(81.2)$ \\
\hline Black & $88(6.4)$ \\
\hline Other & $124(9.0)$ \\
\hline Missing & $47(3.4)$ \\
\hline \multicolumn{2}{|l|}{ Gestational age (weeks): } \\
\hline $24-27$ & $703(51.0)$ \\
\hline $28-31$ & $367(26.7)$ \\
\hline $32-35$ & $130(9.4)$ \\
\hline$\geq 36$ & $177(12.9)$ \\
\hline \multicolumn{2}{|l|}{ Presentation†: } \\
\hline Cephalic & $471(34.2)$ \\
\hline Breech & $327(23.8)$ \\
\hline Other & $77(5.6)$ \\
\hline Missing* & $502(36.5)$ \\
\hline \multicolumn{2}{|l|}{ Mode of deliveryt: } \\
\hline Spontaneous vaginal & $343(24.9)$ \\
\hline Assisted vaginal & $114(8.3)$ \\
\hline Elective caesarean & $74(5.4)$ \\
\hline Emergency caesarean & $442(32.1)$ \\
\hline Other/missing* & $404(29.3)$ \\
\hline \multicolumn{2}{|l|}{ Sex of infantt: } \\
\hline Male & $786(57.1)$ \\
\hline Female & $587(42.6)$ \\
\hline Missing & $4(0.3)$ \\
\hline \multicolumn{2}{|l|}{ Birth weight $(\mathrm{g}) \dagger$ : } \\
\hline Median (IQR) & $970(730-1440)$ \\
\hline Missing & $14(1.0)$ \\
\hline \multicolumn{2}{|l|}{ Cause of death: } \\
\hline Asphyxia, anoxia, or trauma & $242(17.6)$ \\
\hline Immaturity & $735(53.5)$ \\
\hline Infection & $164(11.9)$ \\
\hline Other specific & $187(13.6)$ \\
\hline Injury & $4(0.3)$ \\
\hline Unexplained $\ddagger$ & $39(2.8)$ \\
\hline Missing or unclassifiable & $6(0.4)$ \\
\hline
\end{tabular}

$\mathrm{IQR}=$ interquartile range.

*Recorded only from 1996 onwards; $93.8 \%$ of missing records for mode of delivery and $74.7 \%$ of missing records for presentation were for births before 1996.

†For infant who died; data were not available for the survivor. flncludes in utero and neonatal deaths.

relation to method of delivery in the 121 twin pairs for whom we had this information. When we looked at all causes of perinatal death related to delivery we found no significant difference in those delivered by caesarean section (odds ratio for interaction term $0.9,0.4$ to $1.8, \mathrm{P}=0.7)$. When we confined the analysis to deaths caused by intrapartum anoxia we found an interaction of borderline significance between birth order and caesarean section $(0.4,0.1$ to $1.3, \mathrm{P}=0.1)$. The odds ratio for the second twin was 1.8 (0.9 to 3.6) among those delivered by caesarean section and 4.1 (1.8 to 9.5$)$ among

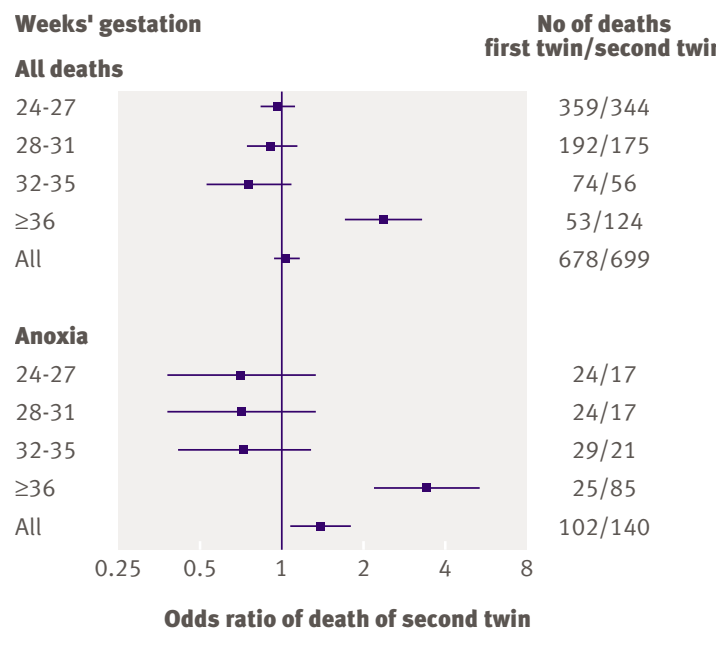

Fig 1| Odds ratio for perinatal death related to delivery of second twin for all causes and deaths caused by anoxia, stratified by gestational age. Numbers of deaths are actual numbers of losses of first and second twins, confined to births where other twin survived

those delivered vaginally (fig 2). There were only 19 twin pairs delivered by planned caesarean section at term where one died and the other survived. Among this group, the odds ratio for any perinatal death of the second twin related to delivery was $1.4(0.6$ to 3.4$)$ and 1.0 ( 0.1 to 7.1$)$ for death caused by anoxia.

\section{Sensitivity analyses}

We performed two sensitivity analyses. Firstly, we repeated the analysis excluding eligible births from 1994 ( $n=178)$, when the dataset was less complete. The results were similar to the main analysis, with a significant interaction between birth order and gestational age $(\mathrm{P}<0.001)$ and no significant association between birth order and the risk of death at preterm gestations but a significantly increased risk of all cause death for the second twin at term $(2.4,1.7$ to $3.5)$ and for death caused by anoxia (3.3, 2.0 to 5.4). Secondly, we repeated the analysis including cases

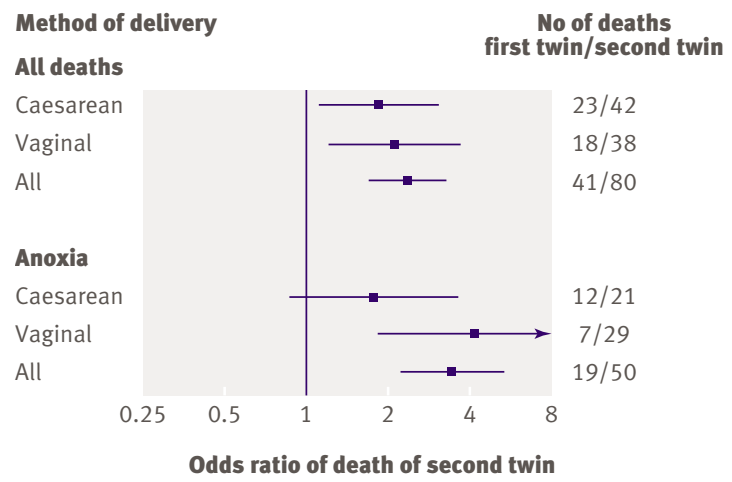

Fig 2 | Odds ratio for perinatal death related to delivery of second twin at term gestation for all deaths and deaths caused by anoxia, stratified by method of delivery. Numbers of deaths are actual numbers of losses of first and second twins, confined to births where the other twin survived (excludes cases with missing record of method of delivery) 
where the baby was stillborn but the timing of death in relation to the onset of labour was documented as "unknown" ( $\mathrm{n}=179)$. The results were similar to the main dataset, with a significant interaction between birth order and gestational age $(\mathrm{P}<0.001)$ and no significant association between birth order and the risk of death at preterm gestations but a significantly increased risk of all cause death for the second twin at term $(2.3,1.8$ to 3.0$)$ and for death caused by anoxia $(3.3,2.1$ to 5.0$)$.

\section{DISCUSSION}

In this retrospective cohort study we found an increased risk of death of second twins compared with first twins born at term in England, Northern Ireland, and Wales, 1994-2003. There was an interaction between the effect of birth order and gestational age. There was no association between birth order and the risk of death among infants born at preterm gestations, but we found a strong association between birth order and the risk of death at term. The interaction between birth order and gestational age is unlikely to be a chance finding. Firstly, it was highly significant $(\mathrm{P}<0.001)$. Secondly, we had previously observed such an interaction in another population, ${ }^{2}$ and the presence of an interaction was a prior hypothesis. Thirdly, it is biologically plausible. The risk of death at term is low and a small absolute risk of complications for the second twin will result in a much greater relative risk of death (when compared with the first twin) than at preterm gestations, where the background risk of death is high for both. ${ }^{2}$ The association between birth order and the risk of perinatal death at term was stronger for deaths attributed to intrapartum anoxia. These findings clearly show an increased risk for death of the second twin delivered at term, principally because of complications of labour and delivery.

\section{Comparison with other research}

A previous observational study found a lower risk of death of either twin with planned caesarean delivery. ${ }^{3}$ Consistent with this, the risk of anoxic death of the second twin was lower among those delivered by caesarean section than those delivered vaginally, although not significantly so. The data source we used was confined to infants who died so we did not know how the surviving twin had been delivered. In some cases where the second twin died after a caesarean delivery, the first twin may have been delivered vaginally. This delivery combination is known to increase the risk of death for the second twin. ${ }^{910}$ It is likely, therefore, that the protective effect of caesarean delivery would be greater than our results suggest. The number of planned caesarean sections in the present analysis was too small to confirm or exclude a significant association between birth order and the risk of perinatal death with this method of delivery.

A large scale study of US birth and death certifications (1995-7) published in 2004 found no significant difference in the risk of neonatal death among second twins. ${ }^{4}$ The analysis, however, had several weaknesses -namely, the failure to use paired statistical comparison of first and second twins, the known shortcomings of the US birth and death certification databases, ${ }^{11} 12$ and stratification by birth weight rather than gestational age. Moreover, other analyses of the same data source suggested an increased risk of death of the second twin related to delivery: the risk of neonatal death of the second twin was lower when both babies were delivered by caesarean section compared with those delivered vaginally. ${ }^{910}$ The US data also lack information on whether death of the infant occurred before or during labour and cannot, therefore, address the effect of birth order on the risk of intrapartum stillbirth. The strengths of our study are the use of more appropriate statistical methods and that data were available for a large number of losses, including detailed information on both the timing and cause of perinatal death.

Our results are consistent with those of several previous studies of birth order and perinatal morbidity. These have shown an increased risk of a depressed five minute Apgar score in the second twin. ${ }^{513} \mathrm{We}$ found no association between birth order and the risk of perinatal death at preterm gestations, whereas other studies have shown an increased risk of fetal distress or morbidity for second twins born preterm. ${ }^{13}$ Our interpretation of these findings is that labour and delivery are associated with risks to the second twin at all gestations. The major determinant of perinatal death at preterm gestations, however, is the degree of prematurity. Hence, a small additional risk to the second twin during vaginal birth has no significant effect on the relative risk of death, except at term. Other studies with data on both twins have identified risk factors for death of the second twin, including discordant birth weights, ${ }^{2}$ delivery by a means other than planned caesarean section, ${ }^{3}$ operative vaginal delivery of the first twin (when compared with spontaneous vaginal delivery of the first twin) ${ }^{14}$ and a prolonged interval between delivery of the first twin and delivery of the second..$^{13}$

The findings of this and other studies suggest that planned caesarean section may be beneficial for all twins. Direct evidence for a protective effect of caesarean section would require a randomised controlled trial, although statistical power might be a problem. ${ }^{2}$ This and previous studies have important lessons for any randomised controlled trial of planned caesarean

\section{WHAT IS ALREADY KNOWN ON THIS TOPIC}

Vaginal delivery of the second twin is recognised as a time of high risk

Recent studies of the effect of birth order on the risk of perinatal death have produced inconsistent results

\section{WHAT THIS STUDY ADDS}

There was no association between birth order and the relative risk of perinatal death related to delivery among preterm twins

At term, the second twin had a greater than twofold risk of perinatal death related to delivery and a greater than threefold risk of death caused by intrapartum anoxia 
section for all twin pregnancies. Inclusion of preterm births may mask a protective effect of caesarean section on perinatal mortality if the principal effect of caesarean section is to reduce the risk of complications for the second twin. Moreover, this and previous studies showed that it is a minority of all perinatal deaths of twins that are related to complications during labour and delivery. Failure to exclude losses that are largely independent of method of delivery, including antepartum stillbirth and deaths caused by congenital abnormality or prematurity, may mask a protective effect of caesarean delivery.

Contributors: GCSS had the original idea and is guarantor. All authors discussed the study design and analytic approach. GCSS and KMF performed the statistical analysis. GCSS drafted the article and all authors contributed to and approved the final version.

Funding: None.

Competing interests: None declared.

Ethical approval: The directors of CEMACH approved the study.

1 Smith GCS. Estimating risks of perinatal death. Am J Obstet Gynecol 2005;192:17-22.

2 Smith GCS, Pell JP, Dobbie R. Birth order, gestational age, and risk of delivery related perinatal death in twins: retrospective cohort study. BMJ 2002;325:1004

3 Smith GCS, Shah I, White IR, Pell JP, Dobbie R. Mode of delivery and the risk of delivery-related perinatal death among twins at term: a retrospective cohort study of 8073 births. BJOG 2005;112:1139-44.
4 Sheay W, Ananth CV, Kinzler WL. Perinatal mortality in first- and second-born twins in the United States. Obstet Gynecol 2004;103:63-70.

5 Thorngren-Jerneck K, Herbst A. Low 5-minute Apgar score: a population-based register study of 1 million term births. Obstet Gynecol 2001;98:65-70.

6 Armson BA, O'Connell C, Persad V, Joseph KS, Young DC, Baskett TF. Determinants of perinatal mortality and serious neonatal morbidity in the second twin. Obstet Gynecol 2006;108:556-64.

7 Hey EN, Lloyd DJ, Wigglesworth IS. Classifying perinatal death: fetal and neonatal factors. Br J Obstet Gynaecol 1986;93:1213-23.

8 Goodman SN, Berlin JA. The use of predicted confidence intervals when planning experiments and the misuse of power when interpreting results. Ann Intern Med 1994:121:200-6.

9 Yang Q, Wen SW, Chen Y, Krewski D, Fung KF, Walker M. Neonatal death and morbidity in vertex-nonvertex second twins according to mode of delivery and birth weight. Am I Obstet Gynecol 2005;192:840-7.

10 Yang Q, Wen SW, Chen Y, Krewski D, Fung KF, Walker M. Neonatal mortality and morbidity in vertex-vertex second twins according to mode of delivery and birth weight. J Perinatol 2006;26:3-10.

11 Lydon-Rochelle MT, Cardenas V, Nelson JL, Tomashek KM, Mueller BA, Easterling TR. Validity of maternal and perinatal risk factors reported on fetal death certificates. Am J Public Health 2005;95:1948-51.

12 Cahill AG, Macones GA. Vital considerations for the use of vital statistics in obstetrical research. Am J Obstet Gynecol 2006:194:909-10.

13 Hartley RS, Hitti J. Birth order and delivery interval: analysis of twin pair perinatal outcomes. J Matern Fetal Neonatal Med 2005;17:375-80.

14 Yang Q, Walker MC, Chen XK, Krewski D, Fung Kee FK, Wen SW. Impacts of operative delivery for the first twin on neonatal outcomes in the second twin. Am J Perinatol 2006;23:381-6.

Accepted: 18 January 2007 